\title{
A spectroscopic analysis of Jupiter-coupled object (52872) Okyrhoe, and TNOs (90482) Orcus and (73480) $2002 \mathrm{PN}_{34}$ ᄎ
}

\author{
F. E. DeMeo ${ }^{1}$, M. A. Barucci ${ }^{1}$, F. Merlin ${ }^{2}$, A. Guilbert-Lepoutre ${ }^{3}$, A. Alvarez-Candal ${ }^{4}$, \\ A. Delsanti ${ }^{5}$, S. Fornasier ${ }^{1,6}$, and C. de Bergh ${ }^{1}$ \\ 1 LESIA, Observatoire de Paris, 5 place Jules Janssen, 92195 Meudon Principal Cedex, France \\ e-mail: francesca.demeo@obspm.fr \\ 2 University of Maryland, College Park, MD 20742, USA \\ 3 UCLA Department of Earth and Space Sciences, 595 Charles E. Young Drive E, Los Angeles, CA 90095, USA \\ 4 ESO, Alonso de Cordova 3107, Vitacura, Casilla 19001, Santiago 19, Chile \\ 5 Laboratoire d'Astrophysique de Marseille, Université de Provence, CNRS, 38 rue Frédéric Joliot-Curie, 13388 Marseille Cedex 13, \\ France \\ ${ }^{6}$ Université de Paris 7 Denis Diderot, Paris, France
}

Received 11 January 2010 / Accepted 19 May 2010

ABSTRACT

\begin{abstract}
Aims. We present new visible and near-infrared photometric measurements and near-infrared spectroscopic measurements for three outer solar system small bodies, the Jupiter-coupled object (52872) Okyrhoe and the TNOs (90482) Orcus and (73480) 2002 PN $_{34}$. Methods. We analyzed their surface compositions by modeling their spectra in the visible and near-infrared wavelength ranges. We then compared this new data with previous measurements of Okyrhoe and Orcus to search for heterogeneity on their surfaces. All observations were performed at the European Southern Observatory $8 \mathrm{~m}$ Very Large Telescope, UT1 and UT4 at the Paranal Observatory in Chile.

Results. We find varying amounts of $\mathrm{H}_{2} \mathrm{O}$ ice among these bodies, Okyrhoe shows no trace of it in our spectrum, 73480 has small amounts, and Orcus has large quantities. While we do clearly see for Orcus that a significant fraction of the $\mathrm{H}_{2} \mathrm{O}$ ice is in crystalline form from the $1.65-\mu \mathrm{m}$ feature, we cannot detect the $2.21-\mu \mathrm{m}$ feature supposedly due to ammonia hydrate, because of the low signalto-noise of the data. We also do not see any indication of ices more volatile than $\mathrm{H}_{2} \mathrm{O}$, such as $\mathrm{CH}_{4}$ or $\mathrm{CO}_{2}$, in the spectrum, so we limit their presence to no more than about $5 \%$ based on the data presented here and on high-quality data from Barucci et al. (2008,
\end{abstract} A\&A, 479, L13).

Key words. techniques: spectroscopic - techniques: photometric - Kuiper belt objects: individual: (52872) Okyrhoe Kuiper belt objects: individual: (90482) Orcus - Kuiper belt objects: individual: (73480) 2002 PN $_{34}$

\section{Introduction}

Transneptunian object (TNO) spectral signatures provide important constraints on surface composition. While most visible spectra are featureless (Lazzarin et al. 2003; Fornasier et al. 2009), many of the TNO surfaces are ice-rich and have clear spectral signatures in the near-infrared. Some TNO near-infrared spectra are largely featureless and only variations in spectral slope allow us to distinguish one from another (Guilbert et al. 2009). Only for the largest and brightest TNOs can spectra be measured with a sufficiently high signal-to-noise ratio necessary for a detailed analysis. For dimmer bodies, a taxonomy of photometric colors of TNOs helps categorize objects with similar characteristics (Barucci et al. 2005a). The four classes are BB, $\mathrm{BR}, \mathrm{IR}$, and RR, spanning from neutral colors to very red with respect to the Sun.

The spectra of small bodies in the outer solar system have been divided into a few compositional groups. The largest bodies contain a significant fraction of volatiles on their surfaces. The signatures of methane are particularly apparent on Pluto, Eris, Makemake, Quaoar, and Sedna, (e.g., Cruikshank et al. 1976;

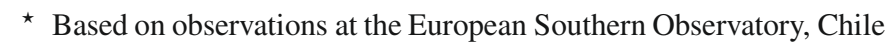
under programs 178.C-0867 and 178.C-0036.
Brown et al. 2005; Licandro et al. 2006b; Schaller \& Brown 2007a; Barucci et al. 2005b). Many TNOs and Centaurs show signatures of $\mathrm{H}_{2} \mathrm{O}$ ice (Barkume et al. 2008; Guilbert et al. 2009). Rare signatures of $\mathrm{NH}_{3}$ (on Charon and Orcus; Buie \& Grundy 2000; Brown \& Calvin 2000; Barucci et al. 2008) and methanol ((5145) Pholus and 2002 VE$_{95}$, Cruikshank et al. 1998; Barucci et al. 2006) have also been detected. Featureless spectra have a wide range of slopes but no distinct absorption bands, thus leaving the surface composition largely unknown.

Further complicating our understanding of these bodies is a process called space weathering, which includes solar wind, cosmic ray, and micrometeorite bombardment, which together alter the chemical and physical properties of surfaces. Laboratory studies have provided insight into how these processes affect material expected to be on the surfaces (Strazzulla \& Palumbo 2001; Strazzulla et al. 2003; Brunetto et al. 2006). Space weathering affects the surfaces of larger, volatile-rich bodies differently than the surfaces of smaller ones. Rejuvenation processes, such as collisions and for larger bodies sublimation and recondensation of ices and possibly cryovolcanism, are in constant competition with the space-weathering aging, process.

Here we present new visible and near-infrared photometric measurements and near-infrared wavelength spectra for the 
Jupiter-coupled object (52872) Okyrhoe, the resonant object (90482) Orcus, and scattered disk object (73480) 2002 PN $_{34}$. Their physical and orbital properties are listed in Table 1. These observations are part of a second Large Program (PI M. A. Barucci) dedicated to investigating the surface properties of Centaurs and transneptunian objects. The program was carried out between 2006 and 2008 using the European Southern Observatory (ESO) Very Large Telescope (VLT). Observational methods for this program included spectroscopy, photometry (colors and lightcurves), and polarimetry to characterize TNOs with the most information available from ground-based studies. For a summary of previously published results see DeMeo et al. (2010). In this work we present observations and reduction in Sect. 2, we describe the modeling procedure in Sect. 3, and we suggest surface compositions for each object in Sect. 4 based on bi-directional reflectance models, and compare the data to previously measured spectra to search for heterogeneity.

\section{Observations and data reduction}

The observations for these three objects were performed at the ESO 8.2-m Very Large Telescope (VLT), unit 1 (UT1) and unit 4 (UT4) at the Paranal Observatory in Chile. (73480) $2002 \mathrm{PN}_{34}$ was observed in service mode on 10 and 12 November 2007, while (90482) Orcus and (52872) Okyrhoe were observed in visitor mode on 3 and 4 February 2008. Photometry data were taken with FORS2 for visible wavelengths and ISAAC for near-infrared. The photometric observation and reduction follow those explained by Perna et al. (2010) for a larger sample of TNOs. We include visible wavelength spectra taken with FORS2 reported in Fornasier et al. (2009), because they have the highest signal-to-noise ratios of all previously published data and were taken during the same observation run as the data presented here. For a list of observational information see Table 2.

\subsection{FORS2}

The instrument FORS2 (Focal Reducer and low dispersion Spectrograph, Appenzeller et al. 1998) on UT1 was used for visible photometry. It is equipped with a mosaic of two $2 \mathrm{k} \times$ 4k MIT CCDs with square $15 \mu \mathrm{m}$ pixels. The broadband $V, R$, $I$ filters are centered at $0.554,0.655$ and $0.768 \mu \mathrm{m}$, respectively.

As explained in DeMeo et al. (2009), the photometric CCD images were reduced and calibrated with a standard method using the MIDAS software package. Master bias and flat-fields from daylight calibrations were used. The instrumental magnitudes were measured using aperture photometry with an integrating radius typically about three times the average seeing, and sky subtraction was performed using an annulus width of 5-10 pixels around each object. The zero point of the telescope, extinction coefficient and color correction terms were computed for each run by a least square fit for the standard stars. Atmospheric extinction was corrected using the standard stars and also stars in the field of the objects. The absolute calibration was obtained through the observation of several Landolt fields (Landolt 1992). The visible and near-infrared photometric results are given in Table 3.

\subsection{ISAAC}

The instrument ISAAC (Infrared Spectrometer and Array Camera, Moorwood et al. 1998) also on UT1 was used for near-infrared photometry and spectroscopy. It was used in short
Table 1. Target information.

\begin{tabular}{|c|c|c|c|}
\hline \multirow[t]{2}{*}{ Parameter } & $(52872)$ & $(73480)$ & (90482) \\
\hline & Okyrhoe & $2002 \mathrm{PN}_{34}$ & Orcus \\
\hline Orbit & Jupiter-Coupled $^{a}$ & Scattered Disk & Resonant (3:2) \\
\hline Albedo $(\%)^{b}$ & $2.49_{-0.55}^{+0.81}$ & $4.25_{-0.65}^{+0.83}$ & $19.72_{-2.76}^{+3.40}$ \\
\hline Diam. (km) & $52.1_{-6.9}^{+6.9}$ & $119.5_{-10.2}^{+10.3}$ & $946.3_{-72.3}^{+74.1}$ \\
\hline$a(\mathrm{AU})^{c}$ & 8.4 & 31.2 & 39.2 \\
\hline$e$ & 0.31 & 0.57 & 0.23 \\
\hline$i(\mathrm{deg})$ & 15.7 & 16.6 & 20.6 \\
\hline Taxonomy $^{d}$ & $\mathrm{BR}$ & $\mathrm{BR}, \mathrm{BB}$ & $\mathrm{BB}$ \\
\hline
\end{tabular}

Notes. ${ }^{(a)}$ Some classify object 52872 as a Centaur, but because its Tisserand parameter $T_{\mathrm{J}}$ is less than $3.05\left(T_{\mathrm{J}}=2.95\right)$ it is "rapidly perturbed by Jupiter" according to Gladman et al. (2008).

(b) From Stansberry et al. (2008).

(c) $a, e$, and $i$ are the orbital elements semi-major axis, eccentricity and inclination, respectively.

(d) Classifications are performed using the taxonomic system designed by Barucci et al. (2005a). The designation comes from Fulchignoni et al. (2008) for Okyrhoe and Orcus and from Perna et al. (2010) for (73480) $2002 \mathrm{PN}_{34}$.

wavelength imaging mode for photometry and short wavelength (1100-1400 nm) low resolution mode (1 arcsec slit) for spectroscopy (resolution of 500). The short wavelength arm is equipped with the $1024 \times 1024$ Hawaii Rockwell array with a pixel size of $18.5 \mu \mathrm{m}$ and a scale of $0.148 \mathrm{arcsec} /$ pixel. For photometry, we observed with the $J, H, K$ s filters, with central wavelength of $1.25,1.65$ and $2.16 \mu \mathrm{m}$, respectively. Multiple shorter expositions were taken rather than a single long one to minimize the noise contribution of sky background which is greater in the near-infrared compared to the visible. The objects were nodded between A and B positions separated by $10^{\prime \prime}$ along the slit for spectroscopic observations.

ISAAC ancillary data reduction frames were produced and applied with the ESO ISAAC data reduction pipeline using the Eclipse package (Devillard 1997) to spectroscopy and imaging data. Electrical ghost signals created by the detector were removed, the offset between odd and even columns of the array were corrected, the master dark file was subtracted and the image was divided by the master flat file. For imaging, the dithered sub-images were sky-corrected and recombined using the IRAF/xdimsum package, and the objects' fluxes were determined following an aperture correction technique described in Delsanti et al. (2004). Similarly to the FORS2 data, the zero point of the telescope and extinction coefficients were computed for each night by a least square fit for the standard stars using faint UKIRT (United Kingdom Infra-Red Telescope) standards (Persson et al. 1998; Hawarden et al. 2001), but color correction terms were set to zero as they are very small. The resulting magnitudes are presented in Table 3 .

For spectroscopy, each pair of spectra was combined using MIDAS as described in Barucci et al. (2002). Xenon and argon lamps were used to perform the wavelength calibration. The spectra were divided by the spectrum of the solar analog star with a similar airmass to obtain the reflectance spectrum for each object.

\subsection{SINFONI}

Near-infrared spectral observations were performed simultaneously in the $H$ and $K$ bands (1400-2500 nm) using SINFONI 
Table 2. Circumstances of spectroscopic observations.

\begin{tabular}{ccccccc}
\hline \hline Object & Instr $^{a}$ & Date & $\begin{array}{c}\mathrm{UT}_{\text {start }} \\
(\mathrm{hh}: \mathrm{mm})\end{array}$ & $\begin{array}{c}T_{\text {exp }} \\
(\mathrm{s})\end{array}$ & Airm & Standard Star (Airm) \\
\hline (52872) Okyrhoe & FORS2 & Feb. 04, 2008 & $06: 33$ & 1800 & 1.14 & SA102 1081 (1.13) \\
$(52872)$ Okyrhoe & ISAAC & Feb. 03, 2008 & $07: 02$ & 1080 & $1.155-1.176$ & HIP 045953 (1.166) \\
$(52872)$ Okyrhoe & SINFONI & Feb. 03, 2008 & $05: 28$ & 7800 & $1.128-1.204$ & SA102 1081 (1.140) \\
$(73480) 2002 \mathrm{PN}_{34}$ & FORS2 & Nov. 10, 2007 & $00: 51$ & 2800 & 1.22 & SA115 271 (1.13) \\
$(73480) 2002 \mathrm{PNN}_{34}$ & SINFONI & Nov. 10, 2007 & $00: 32$ & 6000 & $1.161-1.384$ & SA115 271 (1.167) \\
$(73480) 2002 \mathrm{PN}_{34}$ & SINFONI & Nov. 12, 2007 & $01: 25$ & 3600 & $1.247-1.678$ & SA115 271 (1.296) \\
$(90482)$ Orcus & FORS2 & Feb. 04, 2008 & $05: 25$ & 2400 & 1.07 & SA102 1081(1.11) \\
$(90482)$ Orcus & ISAAC & Feb. 03, 2008 & $05: 36$ & 2880 & $1.058-1.087$ & HIP 045953 (1.060) \\
$(90482)$ Orcus & SINFONI & Feb. 04, 2008 & $03: 54$ & 12600 & $1.058-1.252$ & SA102 1081 (1.135) \\
\hline
\end{tabular}

Notes. ${ }^{(a)}$ FORS2 measures visible wavelength $(0.4-0.8 \mu \mathrm{m})$, visible spectroscopy is from Fornasier et al. (2009), ISAAC measures $J$ band wavelengths $(1-1.4 \mu \mathrm{m})$, SINFONI measures $H+K$ band wavelengths $(1.4-2.4 \mu \mathrm{m})$.

Table 3. Circumstances of and magnitudes from photometric observations.

\begin{tabular}{|c|c|c|c|c|c|c|}
\hline \multirow[t]{2}{*}{ Obj } & (52872) & $(52872)$ & (73480) & (73480) & (90482) & (90482) \\
\hline & Okyrhoe & Okyrhoe & $2002 \mathrm{PN}_{34}$ & $2002 \mathrm{PN}_{34}$ & Orcus & Orcus \\
\hline Date & 3 Feb. 2008 & 4 Feb. 2008 & 10 Nov. 2007 & 10 Nov. 2007 & 3 Feb. 2008 & 4 Feb. 2008 \\
\hline $\mathrm{UT}_{\text {start }}$ & $06: 41$ & $06: 18$ & $00: 36$ & $02: 23$ & $04: 46$ & $05: 04$ \\
\hline$V$ & & $18.63 \pm 0.02$ & $20.68 \pm 0.03$ & & & $19.12 \pm 0.02$ \\
\hline$R$ & & $18.14 \pm 0.02$ & $20.25 \pm 0.05$ & & & $18.73 \pm 0.02$ \\
\hline$I$ & & $17.64 \pm 0.02$ & & & & $18.37 \pm 0.02$ \\
\hline$J$ & $16.84 \pm 0.06$ & & & $19.00 \pm 0.05$ & $17.91 \pm 0.05$ & \\
\hline$H$ & $16.39 \pm 0.07$ & & & $18.49 \pm 0.06$ & $17.72 \pm 0.07$ & \\
\hline$K_{\mathrm{s}}$ & $16.28 \pm 0.05$ & & & $18.29 \pm 0.05$ & $17.89 \pm 0.05$ & \\
\hline$\Delta(\mathrm{AU})^{a}$ & 4.883 & 4.877 & 14.894 & 14.894 & 46.904 & 46.9 \\
\hline$r(\mathrm{AU})$ & 5.800 & 5.800 & 15.344 & 15.344 & 47.807 & 47.807 \\
\hline$\alpha(\mathrm{deg})$ & 3.9 & 3.7 & 3.3 & 3.3 & 0.5 & 0.5 \\
\hline
\end{tabular}

Notes. ${ }^{(a)} \Delta, r$, and $\alpha$ are the topocentric distance, heliocentric distance, and phase angle, respectively.

(Infrared Spectrometer and Array Camera, Eisenhauer et al. 2003; Bonnet et al. 2004). As described by Dumas et al. (2007), SINFONI is an integral field spectrometer with a field-of-view split into 32 image-slitlets which reflect onto small plane mirrors and then re-directed toward the selected grating. The 32 spectra are then re-imaged on a $2048 \times 2048$ pixel Hawaii 2 RG near-infrared detector. The $H+K$ spectral grating has a spectral resolution of 1500 and a plate scale of $0.25^{\prime \prime} /$ pixel. Multiple images were taken in a dithering pattern to allow optimal sky correction.

Reduction was performed using the ESO SINFONI pipeline version 2.0.0. Master darks, master flats, bad pixel maps, and wavemaps were created within the SINFONI pipeline. Xenonargon-krypton lamps were used for wavelength calibration, and as part of the SINFONI pipeline, a wavelength map was computed to derive a direct correspondence between pixel position and wavelength. The single images were then combined and reconstructed into an image-cube of the original field-ofview. The spectra were then extracted from the individual data cubes using QFitsView, the 3D-visualisation tool developed at the Max Planck Institute for Extraterrestrial Physics (MPE) for SINFONI (http://www .mpe.mpg.de/ ott/QFitsView). Within QFitsView, an aperture radius and center were chosen and the flux within that circle is summed per image slice to create the spectrum. Each spectrum was then divided by the solar analog spectrum to remove the solar contribution and telluric features. For more detailed information about the reduction of TNO data from SINFONI see Guilbert et al. (2009).

\subsection{Combining and filtering the spectra}

To combine each spectral part to one continuous spectrum and decrease the resolution (to increase the signal-to-noise ratio), we follow a method similar to that described in Merlin et al. (2009). The ISAAC and SINFONI data are convolved with a kernel of length 30 points and sigma of 5 to reduce the resolution to approximately 50 for ISAAC and 250 for SINFONI data. Each normalized spectrum is multiplied by the reflectance from the corresponding photometric measurement. The spectrum is then normalized to unity (or the visible geometric albedo) at $0.55 \mu \mathrm{m}$. The spectra at reduced resolution are presented in Figs. 1, 3, and 4.

\section{Modeling}

We apply a radiative transfer model introduced by Hapke (1981). We calculate the geometric albedo $A_{r}$ using Eq. (1) (Eq. (44) from Hapke 1981) which is a function of the bihemispherical reflectance for isotropic scatterers, $r_{0}$, (Eq. (34) of Hapke 1981) and the average single-scattering albedo, $w$. Because TNOs are observed at small phase angles we set the phase angle to zero, a good approximation. We use a Henyey-Greenstein function (Henyey \& Greenstein 1941) for the phase function, $P_{0}$. For Orcus and 73480, we set the asymmetry parameter to 0.3 for the purposes of this model which corresponds to a forwardscattering surface. Because Okyrhoe's albedo is so low, we had to set the asymmetry parameter to 0.35 to adequately reproduce 
its spectrum. We set the backscattering function to a fixed value, $B_{0}=0.67$, a value proposed by Verbiscer \& Helfenstein (1998) for icy surfaces. We use the albedo as a constraint when modeling our spectra by normalizing the spectra at $0.55 \mu \mathrm{m}$ to the visible albedo reported in Stansberry et al. (2008). To model their surfaces we use optical constants derived from laboratory spectra taken under similar conditions as those found in the outer Solar System. The best-fit model for each object is determined by minimizing the $\chi^{2}$ value which quantifies the difference between the data and the model. In the case for these models, the $\chi^{2}$ value is calculated based on a weighting of the spectrum, so that the fit near important features is considered much more important than the fit in the telluric regions for example.

$A_{r}=\frac{1}{2} r_{0}\left(1+\frac{1}{3} r_{0}\right)+\frac{w}{8}\left(\left(1+B_{0}\right) P_{0}-1\right)$.

We use an intimate mixture of materials, meaning that a photon interacts with grains of more than one composition before being reflected off the surface. Surfaces are likely, however, to have some combination of horizontally separated "patches", vertical layers, and intimate mixtures of grains. We use optical constants of $\mathrm{H}_{2} \mathrm{O}$ ice in both crystalline and amorphous form (at $40 \mathrm{~K}$ and 38 K from Grundy \& Schmitt 1998; and Schmitt et al. 1998, respectively), amorphous carbon (Zubko et al. 1996), and Titan and Triton tholin (Khare et al. 1984, 1993). Carbon and tholins do not have unique, identifiable absorption bands, and thus their presence on these surfaces is not definite. They are used as representative material that aid in fitting the low albedo and red slope of the spectra, because optical constants are available for these materials. However they could be replaced with different dark or red materials, such as other organics.

For Orcus, we model the spectrum with ammonia hydrate (Roush, personal communication), as well as $\mathrm{CH}_{4}$ and $\mathrm{CO}_{2}$ (both from Quirico \& Schmitt 1997) to place constraints on their abundance. We also artificially create a near-infrared-absorbing "blue" material, similar to that added in the models of Barucci et al. (2008), to reproduce the spectral behavior. The need for this component is demonstrated in Sect. 4. The quantities and grain sizes for each component in each model are given in Table 4, and the best model for each object is plotted in Figs. 1, 3, and 4.

\section{Discussion}

Physical and dynamical properties of these three outer Solar System objects are given in Table 1. We discuss each spectrum and the models in detail in the following subsections and compare our data for both Okyrhoe and Orcus to previously published work.

\section{1. (52872) Okyrhoe}

Okyrhoe, discovered in 1998, is often considered a Centaur because of its low semi-major axis and perihelion (with respect to TNOs), but is dynamically classified as a Jupiter-Coupled object by Gladman et al. (2008) because it has a Jupiter-Tisserand parameter $\left(T_{\mathrm{J}}\right)$ less than 3.05 . It has a diameter of around $50 \mathrm{~km}$ and a very low albedo near $2.5 \%$ (Stansberry et al. 2008).

Okyrhoe was first observed in visible wavelengths by Lazzarin et al. (2003). The spectrum had no absorption features and a slope of $11.8 \pm 0.2\left(\% / 10^{3} \AA\right)$. The visible spectrum used for modeling in this work, presented by Fornasier et al. (2009), is also featureless, but has a slightly higher slope of $13.4 \pm 0.6$ $\left(\% / 10^{3} \AA\right)$, however, they are still similar within a 2 sigma level
Table 4. Best-fit models.

\begin{tabular}{|c|c|c|c|}
\hline $\begin{array}{l}\text { Object } \\
\text { model }\end{array}$ & $\begin{array}{c}\text { Amount } \\
(\%)\end{array}$ & $\begin{array}{l}\text { Grain size } \\
\qquad(\mu \mathrm{m})\end{array}$ & Material \\
\hline$(52872)$ & 71 & 10 & Amorphous carbon \\
\hline Okyrhoe & 26 & 10 & Triton tholin \\
\hline$\chi^{2}=2.03$ & 3 & 10 & Titan tholin \\
\hline$(73480)$ & 55 & 10 & Amorphous carbon \\
\hline $2002 \mathrm{PN}_{34}$ & 34 & 10 & Triton tholin \\
\hline$\chi^{2}=181.21$ & 11 & 40 & Amorphous $\mathrm{H}_{2} \mathrm{O}$ \\
\hline (90482) & 50 & 15 & Crystalline $\mathrm{H}_{2} \mathrm{O}$ \\
\hline Orcus & 23 & 15 & Amorphous $\mathrm{H}_{2} \mathrm{O}$ \\
\hline Model 1 & 19 & 10 & Amorphous carbon \\
\hline$\chi^{2}=200.06$ & 8 & 10 & Triton tholin \\
\hline (90482) & 50 & 15 & Crystalline $\mathrm{H}_{2} \mathrm{O}$ \\
\hline Orcus & 22.5 & 15 & Amorphous $\mathrm{H}_{2} \mathrm{O}$ \\
\hline Model 2 & 20.5 & 10 & "Blue" component \\
\hline \multirow{3}{*}{$\chi^{2}=136.49$} & 5 & 10 & Triton tholin \\
\hline & 2 & 10 & Titan tholin \\
\hline & 35.5 & 10 & Amorphous $\mathrm{H}_{2} \mathrm{O}$ \\
\hline (90482) & 30 & 10 & Crystalline $\mathrm{H}_{2} \mathrm{O}$ \\
\hline Orcus & 20.5 & 10 & "Blue" component \\
\hline Model 3 & 7 & 100 & Ammonia hydrate \\
\hline \multirow{2}{*}{$\chi^{2}=137.56$} & 5 & 10 & Triton tholin \\
\hline & 2 & 10 & Titan tholin \\
\hline
\end{tabular}

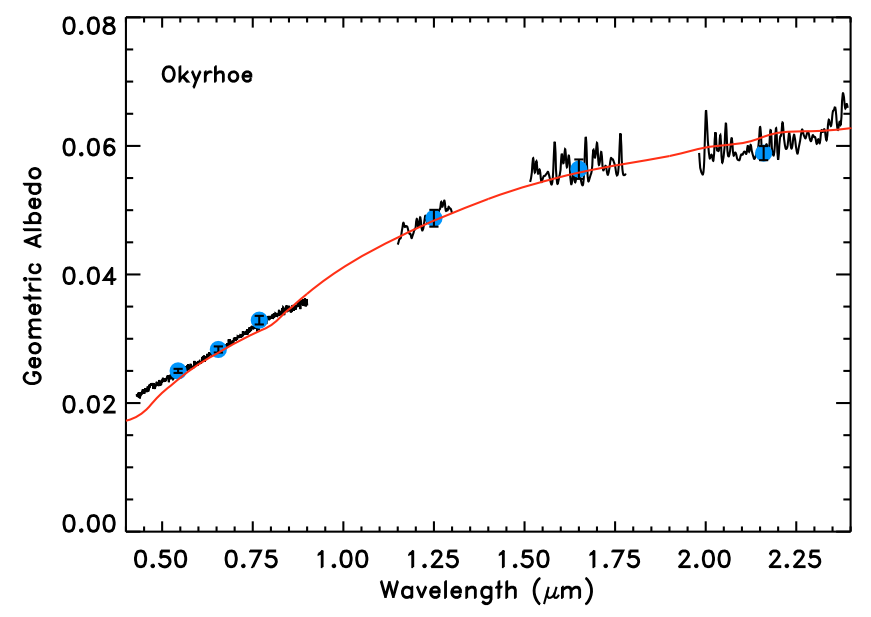

Fig. 1. The spectrum of Okyrhoe and the best model. Blue circles mark reflectance values converted from photometric colors, and the errors are marked in black (but are smaller than the size of the circle). The best fit model (red) includes amorphous carbon, Triton tholin and Titan tholin.

of the uncertainties. Furthermore, Lazzarin et al. (2003) did not consider systematic uncertainties due to the solar analogue stars in the determination of the slope uncertainties as was done by Fornasier et al. (2009).

The first near-infrared spectrum of Okyrhoe was modeled by Dotto et al. (2003). They find that including a small amount of $\mathrm{H}_{2} \mathrm{O}$ ice improves the fit to the data. Their best model was a spatially segregated combination of kerogen (97\%), olivine (1\%) and $\mathrm{H}_{2} \mathrm{O}$ ice (2\%). The high percentage of kerogen was used to reproduce the slope of the visible spectrum while maintaining the flatness in the near-infrared. Albedo was not used as a constraint in their models, although their model did result in a low 


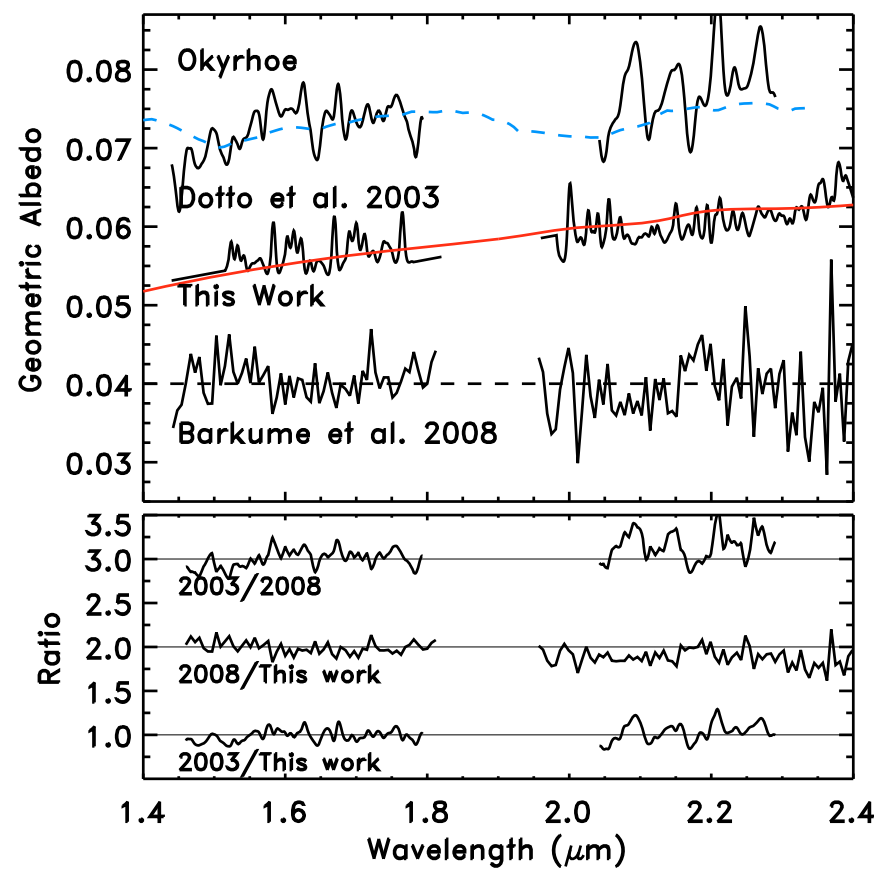

Fig. 2. The spectra of Okyrhoe from Barkume et al. (2008), this work, and Dotto et al. (2003) (solid black) from bottom to top. We plot our model (solid red) and the model of Dotto et al. (dashed blue). Both data and the model from Dotto et al. have been normalized to 0.025 at 0.55 microns and were shifted vertically by 0.035 for clarity. The data from Barkume et al. has been arbitrarily normalized to 0.04 around $1.5 \mu \mathrm{m}$, a horizontal line (dashed black) at 0.04 is drawn to highlight the flatness of the Barkume et al. spectrum.

albedo of 3\%. Barkume et al. (2008) detected $\mathrm{H}_{2} \mathrm{O}$ ice between the 1 and 3 sigma level on Okyrhoe with a total fraction of 0.11 with an error of almost the same value.

Our best model of Okyrhoe's spectrum includes $71 \%$ amorphous carbon, $26 \%$ Triton tholin, and 3\% Titan tholin, all at grain sizes of $10 \mu \mathrm{m}$. The carbon lowered the albedo, the Triton tholin reddened the spectrum, and small amounts of Titan tholin were useful to reproduce the general shape of the visible spectrum. While the data from Dotto et al. (2003) and Barkume et al. (2008) suggest the possible presence of very small amounts of $\mathrm{H}_{2} \mathrm{O}$, our data does not. This suggests that either there is no $\mathrm{H}_{2} \mathrm{O}$ on the surface of Okyrhoe, or possibly there is a trace amount of $\mathrm{H}_{2} \mathrm{O}$ ice that is unevenly distributed across the surface. Further observations are necessary to constrain the possible presence of $\mathrm{H}_{2} \mathrm{O}$ on parts of the surface.

In Fig. 2 we compare our spectra and models to those of previous work. The bottom panel shows the ratio of these spectra to investigate potential differences. The relative reflectance in the near-infrared with respect to the visible (not shown in the figure) is significantly lower for the Dotto et al. (2003) data compared to ours. While there are many possible explanations for this difference, it is likely a problem with the photometric calibration in previous work, but could represent differences in the location on the surface. We calculate the near-infrared slope (\%/1000 $)$ over the range of 1.45 to 2.35 microns excluding the telluric region near 1.8 microns, and include 0.5 to the error for uncertainties of the standard stars. The slope values we find are $4.1 \pm 1.0$, $-0.6 \pm 1.0$, and $2.9 \pm 0.6$ for the data from Dotto et al., Barkume et al., and this work, respectively. However, the real error is likely larger than the formal error as was found for the model fits from Barkume et al. (2008). Also, there is an additional error

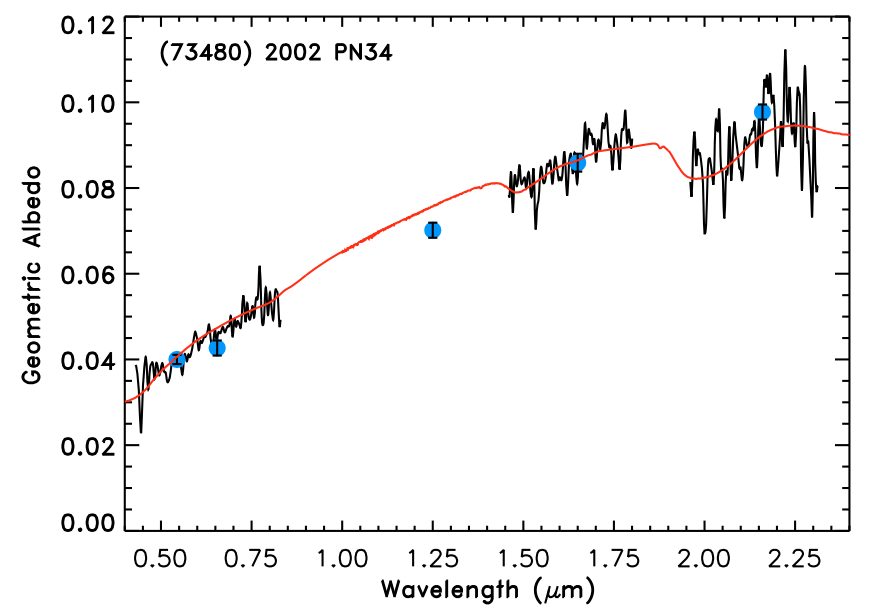

Fig. 3. The spectrum of 73480 (black) with photometric points and errors (blue and black) and the model (red) overlaid. The model includes amorphous carbon, Triton tholin, and amorphous $\mathrm{H}_{2} \mathrm{O}$.

due to the non-simultaneous ( $H$ and $K$ spectra were taken on different nights) observations for the Dotto et al. data. When considering all the sources of error in the measurements, we cannot detect a significant difference in the slopes of these spectra.

\section{2. (73480) $2002 \mathrm{PN}_{34}$}

73480 is a scattered disk object with a diameter of $\sim 120 \mathrm{~km}$ and a low albedo of about $4.25 \%$ (Stansberry et al. 2008). We present here the first near-infrared spectrum of (73480) $2002 \mathrm{PN}_{34}$. The visible slope, published by Fornasier et al. (2009), has a moderately red slope of $15.8 \pm 0.7\left(\% / 10^{3} \AA\right)$. Because these observations were done in service mode, the schedule did not allow us to obtain a spectrum in the $J$-band.

Our best fit model of the spectrum (shown in Fig. 3) includes $55 \%(10 \mu \mathrm{m})$ amorphous carbon to match the low albedo, $34 \%(10 \mu \mathrm{m})$ Triton tholin to create the overall red slope of the spectrum, and $11 \%(40 \mu \mathrm{m})$ amorphous $\mathrm{H}_{2} \mathrm{O}$ ice to reproduce the $2-\mu \mathrm{m}$ feature seen as the drop in albedo before and after the telluric interference between 1.8 and 2 microns. While the body is dark, it does appear to have a measurable amount of $\mathrm{H}_{2} \mathrm{O}$ on the surface. The model generally provides a good fit, but it overestimates the albedo in the $J$ band compared to the single data point from photometric measurements.

\section{3. (90482) Orcus}

Orcus is a Plutino, in the 3:2 resonance with Neptune, discovered in 2004. It is one of the largest bodies, with a diameter of $\sim 950 \mathrm{~km}$ and is one of the brighter minor bodies in the distant Solar System with an albedo of $\sim 19.7 \%$ Stansberry et al. (2008). Fornasier et al. (2004) reported the first visible and near-infrared spectrum of Orcus taken just days after its discovery. Because no albedo measurements were available at the time, they created a low albedo (4\%) and a high albedo (10\%) model. Their low albedo model was created from a geographic mixture of $38 \%$ kerogen, $60 \%$ amorphous carbon and $2 \% \mathrm{H}_{2} \mathrm{O}$ ice. The high albedo model was created from $4 \%$ Titan tholin, $85 \%$ amorphous carbon, and $11 \% \mathrm{H}_{2} \mathrm{O}$ ice. The visible slope they calculated is same as for the spectrum modeled in this work within the errors $(1.8 \% \pm 0.2 \%$ and $1.6 \% \pm 0.6 \%$, respectively, from Fornasier et al. 2004, 2009). 
Orcus' spectrum was revisited by de Bergh et al. (2005). They note the uniqueness of the spectrum due to its nearly neutral-sloped visible spectrum and its relatively strong $\mathrm{H}_{2} \mathrm{O}$ absorptions reminiscent of Charon. Trujillo et al. (2005) modeled a spectrum of Orcus with $\mathrm{H}_{2} \mathrm{O}$ and then calculated the residual by subtracting the model and compared it with $\mathrm{CH}_{4}$ and found a detection within a $3 \sigma$ limit. They found the maximum best-fit surface fraction of $\mathrm{H}_{2} \mathrm{O}$ ice is $50 \% \pm 16 \%$.

A feature near 2.2 microns with a depth of $10 \pm 3 \%$ was first detected by Barucci et al. (2008) in very good signal-tonoise data which could be attributed to either hydrated $\mathrm{NH}_{3}$ or methane. They detected the 1.65-micron feature indicating the presence of $\mathrm{H}_{2} \mathrm{O}$ ice in the crystalline form, and modeled the spectrum with $32 \% \mathrm{H}_{2} \mathrm{O}$ ice (including both crystalline and amorphous), $5 \%$ ammonia diluted in $\mathrm{H}_{2} \mathrm{O}$ ice, and $63 \%$ of a neutrally absorbing blue component to reproduce the overall blue trend in the near-infrared wavelengths. They found that $\mathrm{NH}_{3}$ is likely the main component creating the absorption at $2.2 \mathrm{mi}$ crons, although small amounts of methane cannot be excluded. This feature was also tentatively reported at $2.23 \mu \mathrm{m}$ in an abstract by Cook et al. (2007a). They suggested it could be due to a mixture of pure ammonia ice and ammonia hydrate.

Orcus has a spectrum quite similar to those of Charon and Quaoar in the near-infrared because of the strong signatures of $\mathrm{H}_{2} \mathrm{O}$ crystalline ice and the $2.21 \mu \mathrm{m}$ feature. On Quaoar, the feature at 2.21 microns is expected to be due to methane (Schaller \& Brown 2007a; Dalle Ore et al. 2009) because of other weak features detected in the spectrum. On Charon, however, the lack of other detectable features rules out methane as the primary source of the 2.21-micron band (Cook et al. 2007b).

Both the presence of $\mathrm{H}_{2} \mathrm{O}$ ice in the crystalline form as well as $\mathrm{NH}_{3}$ merit explanation. Crystalline $\mathrm{H}_{2} \mathrm{O}$ ice was previously expected to be amorphized by space weathering processes over the age of the Solar System (Kouchi \& Kuroda 1990), but recent experiments (Mastrapa \& Brown 2006; Zheng et al. 2008), have shown that the $1.65-\mu \mathrm{m}$ band strength decreases when irradiated, but is still present, and that thermal recrystallization is the dominant process at temperatures greater than about $40 \mathrm{~K}$. The amorphization and crystallization processes are discussed in detail in Gil-Hutton et al. (2009). $\mathrm{NH}_{3}$ is expected to be quickly destroyed by irradiation as well (Strazzulla \& Palumbo 1998; Cooper et al. 2003). Proposed mechanisms to maintain $\mathrm{H}_{2} \mathrm{O}$ in crystalline form and resupply $\mathrm{NH}_{3}$ to the surface include cryovolcanism, impact gardening, and solid-state greenhouse or convection, with cryovolcanism being the favored mechanism (Cook et al. 2007b). Another possibility is that irradiation is not as efficient as we expect for $\mathrm{NH}_{3}$, as was found for crystalline $\mathrm{H}_{2} \mathrm{O}$.

In Fig. 5 we plot the near-infrared spectra of Orcus from this work and from de Bergh et al. (2005), and Barucci et al. (2008). The spectrum by de Bergh et al. (2005) has a higher (median percent difference between the ratioed spectra of $18 \pm 10 \%$ ) overall reflectance in the $K$ band than more recent data. Their $H$ and $K$ spectra, however, were not taken simultaneously with the same instrument creating an additional calibration error. Furthermore, the spectra were taken during different nights and so they could have observed different parts of the surface and had different observing conditions. Because the difference in reflectance between our data and the de Bergh et al. data is consistent over the entire $K$ range, the most likely explanation is the photometric calibration, which emphasizes the importance of observing simultaneously over an entire wavelength range. Our spectrum is of lower quality than that presented in Barucci et al. (2008), and we do not detect a clear feature at

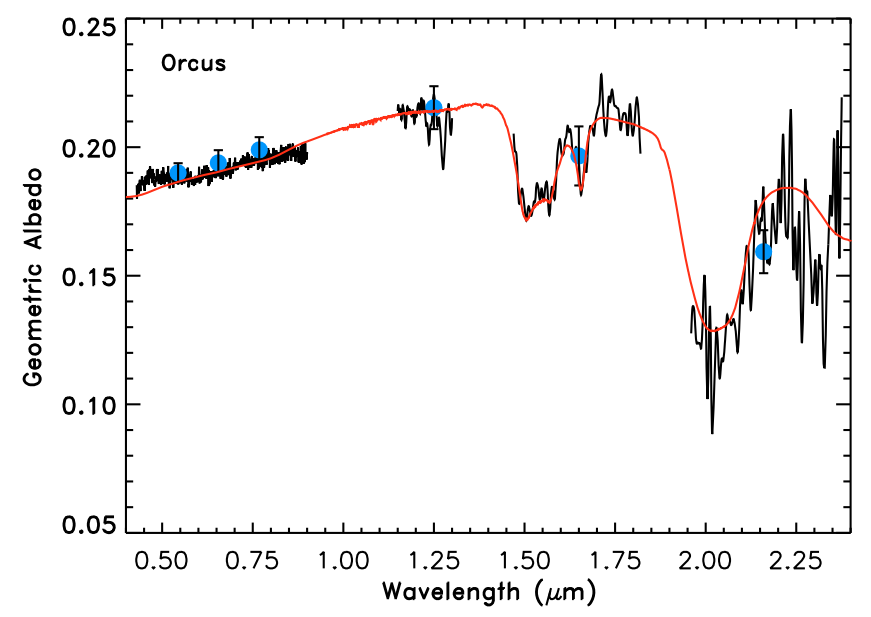

Fig. 4. The spectrum of Orcus (black) with photometric points and errors (blue and black) and the model (red) overlaid. The model includes an artificially created absorbing blue component (see text), tholin (Titan and Triton), and pure $\mathrm{H}_{2} \mathrm{O}$ ice (amorphous and crystalline).

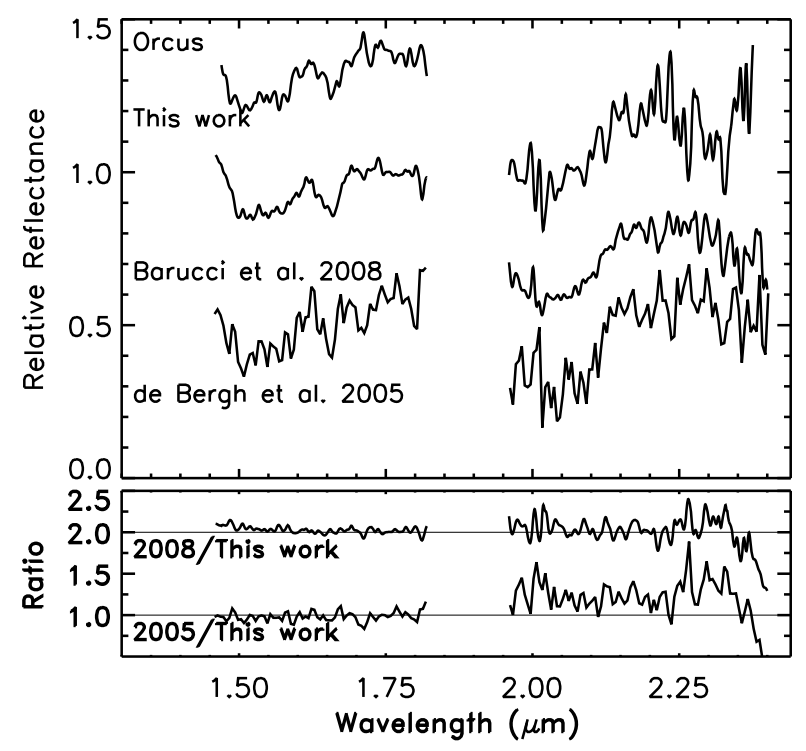

Fig. 5. Top: the near-infrared spectra of Orcus from this and previous work. The data are from de Bergh et al. (2005), Barucci et al. (2008), and this work from bottom to top. The spectra are normalized to 1 at $1.75 \mu \mathrm{m}$, a fairly flat region of the spectrum, and are shifted vertically by 0.4 for clarity. Bottom: the ratio of these previously published spectra to this work.

$\sim 2.2 \mu \mathrm{m}$. To be consistent with the models performed by Barucci et al. (2008), we also present a model of the data with ammonia hydrate, shown in Fig. 6. In our data, any detection is masked by a stronger absorption at wavelengths slightly smaller than 2.2 microns that does not match the location of ammonia, methane or any other known material expected to be on the surface. We suspect this feature is due to noise.

We create three models of Orcus' spectrum shown in Fig. 6. Table 4 lists the concentration of each component used for each model, as well as the $\chi^{2}$ value indicating how well the model fits the data. Model 1 uses amorphous carbon as the absorbing component. It is clear that there is some material on the surface not included in the model that absorbs heavily past $1.7 \mathrm{mi}-$ crons as seen by the large mismatch in the model's albedo. Model 2 provides a better fit; this model is shown over the entire wavelength range in Fig. 4. It includes an artificially created 
F. E. DeMeo et al.: Spectroscopy of three outer solar system small bodies

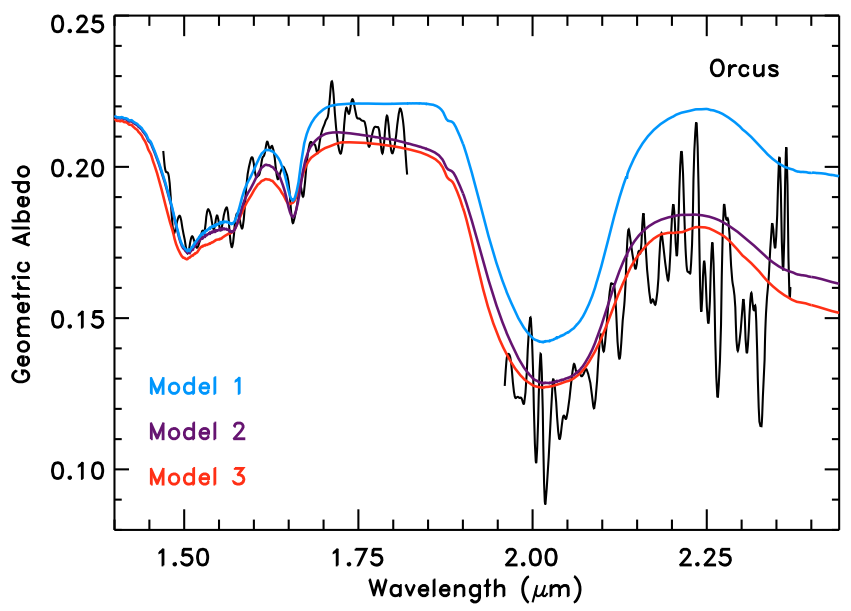

Fig. 6. Three models compared to Orcus' spectrum at reduced resolution. Model 1 (blue) has no blue component and cannot adequately match the spectrum past 2 microns. Model 2 (purple) contains only pure crystalline and amorphous forms of $\mathrm{H}_{2} \mathrm{O}$ ice, while Model 3 (red) includes $100 \mu \mathrm{m}$ grains of $7 \%$ hydrated ammonia in $\mathrm{H}_{2} \mathrm{O}$. See Table 4 for the quantities of each component in each model and the $\chi^{2}$ value indicating goodness-of-fit.

absorbing blue component to account for the lower albedo at longer wavelengths. A similar blue component was used in models by Barucci et al. (2008). These first two models (model 1 and 2) include about $50 \%$ crystalline $\mathrm{H}_{2} \mathrm{O}$ and $23 \%$ amorphous $\mathrm{H}_{2} \mathrm{O}$. Small amounts of tholins were used to reproduce the red slope in the visible wavelengths. Model 3 includes 7\% ammonia hydrate with a grain size of $100 \mu \mathrm{m}$. The quality of our data, however, is not great enough to detect the 2.21-micron feature seen by Barucci et al. (2008). Model 1 is clearly a much poorer fit to the data which is reflected by the larger $\chi^{2}$ value. Model 2 and 3 have very similar fits and similar $\chi^{2}$ values. The addition of large-grain $\mathrm{NH}_{3}$ to the model slightly widens the 2-micron feature, creates a small absorption near 2.2 microns, and reduces the overall albedo. We reduced the grain size of the $\mathrm{H}_{2} \mathrm{O}$ grains for this model to compensate for the darkening caused by the large-grain $\mathrm{NH}_{3}$.

It is evident that we include significantly more $\mathrm{H}_{2} \mathrm{O}$ in our models than in previous work. In Fornasier et al. (2004) the albedo was unknown at the time and so they were modeling a much darker surface. The blue component in our models is more heavily absorbing at longer wavelengths than that used by Barucci et al. (2008), which explains part of the difference between our models and theirs. The choice of modeling parameters could also affect the concentration of each component. If we had chosen a lower cosine asymmetry parameter, we could have recreated Orcus' spectrum just as adequately. This would involve including more absorbing material (carbon or the blue component) and less $\mathrm{H}_{2} \mathrm{O}$ ice to lower the albedo and weaken the bands.

\subsection{Limits on the presence of $\mathrm{CH}_{4}$ and $\mathrm{CO}_{2}$ on Orcus}

Several atmospheric escape models have been used to explain the loss of volatiles on small bodies in the outer Solar System. Schaller \& Brown (2007b) calculate a lower limit to the flux loss by calculating the Jeans escape and ignoring the effects of hydrodynamic escape, which is not as easily constrained. They show that for the size and temperature of Orcus, all volatiles should be lost. Another model was performed by Levi \& Podolak (2009) who show that TNO atmospheres can behave in a way analogous

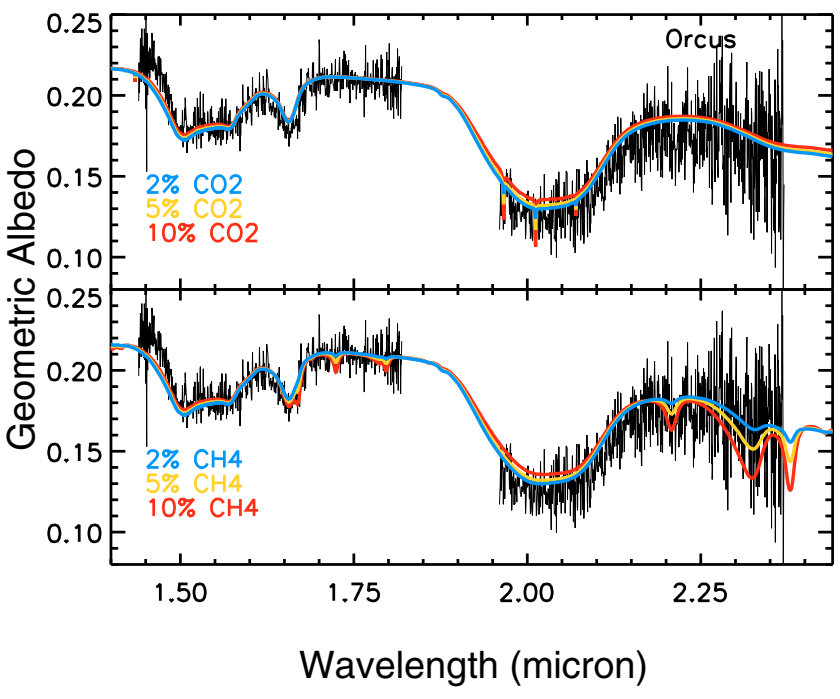

Fig. 7. Comparison of models containing $\mathrm{CH}_{4}$ and $\mathrm{CO}_{2}$ to the full resolution data from Barucci et al. (2008). Top: models with 2, 5, and $10 \% \mathrm{CO}_{2}$ (blue, orange, red). The three narrow bands are located near $2 \mu \mathrm{m}$. Bottom: models with 2, 5, and $10 \% \mathrm{CH}_{4}$ (blue, orange, red).

to coronal expansion using Parker's theory (Parker 1963). They use this theory to explain the absence of $\mathrm{CH}_{4}$ on smaller TNOs and constrain the maximum temperature for retaining methane to $80 \pm 3<T<90_{-12}^{+32}$. A similar model could also be used to predict the presence or absence of other molecules such as $\mathrm{N}_{2}, \mathrm{CO}$, and $\mathrm{CO}_{2}$ on TNOs based on their density, radius, and surface temperature. These three volatiles could possibly be present on the surface of Orcus (Levi \& Podolak 2009, Levi, pers. comm.). $\mathrm{N}_{2}$ and $\mathrm{CH}_{4}$ have been detected on the largest TNOs (e.g., Owen et al. 1993; Licandro et al. 2006a,b; Tegler et al. 2008; Merlin et al. 2009). Because of the intermediate size of Orcus determining the presence or lack of volatiles on its surface provides important constraints for volatile retention. The absorption band for $\mathrm{N}_{2}$ is in a region of $\mathrm{H}_{2} \mathrm{O}$ absorption and is easily detectable only for large grain sizes. The main $\mathrm{CO}$ band is at a long wavelength where the quality of the data is low. We therefore seek to constrain the abundance of $\mathrm{CH}_{4}$ and $\mathrm{CO}_{2}$.

Detecting $\mathrm{CO}_{2}$ on TNOs is particularly difficult because the three strong absorption bands located at 1.966, 2.012, and $2.071 \mu \mathrm{m}$ are very narrow. At high resolution, the signal-to-noise ratio of the data is very low, but at low resolution the thin bands would be lost thus preventing detection. Starting with the composition from Model 2, we create models including 2, 5, and $10 \% \mathrm{CO}_{2}$ (reducing the amount of amorphous $\mathrm{H}_{2} \mathrm{O}$ accordingly) to compare band strengths with the Barucci et al. (2008) data that has the highest signal-to-noise ratio and, like the data from this work, was taken with SINFONI simultaneously in the $H$ and $K$ bands with a resolution of 1500 . We focus only on the $H$ and $K$ band region where the strongest absorption bands are located. In Fig. 7, we can see that by adding $\mathrm{CO}_{2}$, apart from the three narrow bands, the continuum is not significantly affected. The $\chi^{2}$ value of the model fits to the Barucci et al. data as well as the band depths of the strongest $\mathrm{CO}_{2}$ band are given in Table 5. The three $\mathrm{CO}_{2}$ band depths with respect to the continuum when $10 \% \mathrm{CO}_{2}$ is included are 18,21 , and $9 \%$, respectively for the three bands. The noise in the full resolution data in this work at these wavelengths is $\pm 17 \%$, so the data from this work could accommodate nearly $10 \% \mathrm{CO}_{2}$ within the noise. The spectrum from Barucci et al. (2008) has a higher signal-to-noise ratio, and the noise in the data is $\pm 10 \%$ of the median value in 
Table 5. Characteristics of Orcus models including volatiles.

\begin{tabular}{rcc}
\hline \hline Model & Reduced $\chi^{2 a}$ & Depth $(\%)^{b}$ \\
\hline Model 2 & 72 & - \\
$2 \% \mathrm{CO}_{2}$ & 73 & 5 \\
$5 \% \mathrm{CO}_{2}$ & 74 & 11 \\
$10 \% \mathrm{CO}_{2}$ & 76 & 21 \\
$2 \% \mathrm{CH}_{4}$ & 72 & 2 \\
$5 \% \mathrm{CH}_{4}$ & 73 & 5 \\
$10 \% \mathrm{CH}_{4}$ & 80 & 10 \\
\hline
\end{tabular}

Notes. ${ }^{(a)}$ The $\chi^{2}$ is weighted to give greater importance to locations where absorption bands exist $\left(\mathrm{H}_{2} \mathrm{O}\right.$ bands) and lower importance to regions affected by telluric absorption. The value compares the model to the near-infrared data from Barucci et al. (2008). ${ }^{(b)}$ The percentage depth of the strongest $\mathrm{CO}_{2}$ band at $2.012 \mu \mathrm{m}$ and the $\mathrm{CH}_{4}$ band at $\sim 2.2 \mu \mathrm{m}$ relative to the continuum for each model.

this region. In the model with $5 \% \mathrm{CO}_{2}$, the strongest band has a depth of $11 \%$, so their data limit the quantity of $\mathrm{CO}_{2}$ on the surface to a maximum of about $5 \%$.

$\mathrm{CH}_{4}$ should be easier to detect because it has wider and stronger bands than $\mathrm{CO}_{2}$ located at 1.670, 1.724, 1.797, 2.208, 2.324, and $2.379 \mu \mathrm{m}$ (Quirico \& Schmitt 1997). While the last two bands are the strongest, it is the $2.208 \mu \mathrm{m}$ feature that is easiest to identify because it lies in a region relatively free of $\mathrm{H}_{2} \mathrm{O}$ absorption. However, this feature is in nearly the same location as $\mathrm{NH}_{3}$ absorption. While the $\sim 2.21-\mu \mathrm{m}$ feature seen on Charon is thought to be due to $\mathrm{NH}_{3}$ due to the lack of other features (Cook et al. 2007b), on Quaoar the presence of other weak features suggests the presence of $\mathrm{CH}_{4}$ instead (Schaller \& Brown 2007a; Dalle Ore et al. 2009). For models of 5 and 10\% methane, we calculate a $2.21-\mu \mathrm{m}$ band depth of 5 and $10 \%$, respectively. The noise in the data in this region with respect to the median value (calculated from $2.17-2.21 \mu \mathrm{m}$ ) is $\pm 20 \%$ and $9 \%$ in the full-resolution data from this work and Barucci et al., respectively. Since this feature is much wider than the narrow $\mathrm{CO}_{2}$ features, however, the feature may be more easily detected when reducing the resolution to increase the signal-to-noise ratio. The noise in the data from Barucci et al. is $3 \%$ when the resolution is reduced to 250 . The models with varying amounts of pure $\mathrm{CH}_{4}$ are compared to Barucci et al. (2008) in Fig. 7. The $\chi^{2}$ value of the fit including $2 \%$ methane is nearly identical to that for Model 2 (see Table 5), while the values for the 5 and $10 \%$ models become progressively higher. For amounts of around $5 \%$ or less of $\mathrm{CH}_{4}$ the three methane features between 1.6-1.8 $\mu \mathrm{m}$ are very weak even though there is a distinct $2.21-\mu \mathrm{m}$ feature. This raises the possibility that methane could account for the feature seen by Barucci et al. (2008) even if no other features are seen in the data.

\section{Conclusion}

We modeled the spectra of three minor bodies in the outer Solar System, (52872) Okyrhoe, (90482) Orcus, and (73480) $2002 \mathrm{PN}_{34}$, and compared the spectra with those reported in previous work for Okyrhoe and Orcus. Large amounts of amorphous carbon were needed in the model to lower the albedo, although other dark, neutrally absorbing materials could be adequate substitutes. Titan and Triton tholins were important to recreate the red slope of the visible spectrum. Similarly to the carbon, tholins could be replaced with another material that displays red behavior at visible wavelengths.
We found varying amounts of $\mathrm{H}_{2} \mathrm{O}$ ice among these bodies, Okyrhoe had no traces in our spectrum, 73480 had small amounts, and Orcus had large quantities. Previously published spectra of Okyrhoe by Dotto et al. (2003) and Barkume et al. (2008) suggested the possibility of trace amounts of $\mathrm{H}_{2} \mathrm{O}$ ice which could indicate that this minor component, if present, is unevenly distributed across the surface. Further observations are necessary to constrain the possible presence of $\mathrm{H}_{2} \mathrm{O}$ on parts of the surface. The spectrum of Orcus presented here is of lower quality than that presented by Barucci et al. (2008). While we did clearly see that $\mathrm{H}_{2} \mathrm{O}$ ice is in crystalline form from the $1.65-\mu \mathrm{m}$ feature, we could not detect the $2.21-\mu \mathrm{m}$ feature suggested to be due to ammonia hydrate because of the low quality of the data. We also did not see any indication of $\mathrm{CH}_{4}$ or $\mathrm{CO}_{2}$ in the spectrum, so we limited their presence to no more than about $5 \%$.

Acknowledgements. We thank E. Quirico for providing optical constants that made the modeling possible and E. Dotto for providing spectra of Okyrhoe. We are grateful to an anonymous reviewer for very helpful comments. F.E.D. was supported by the grant Excellence Eiffel Égide from the French government.

\section{References}

Appenzeller, I., Fricke, K., Fürtig, W., et al. 1998, The Messenger, 94, 1 Barkume, K. M., Brown, M. E., \& Schaller, E. L. 2008, AJ, 135, 55 Barucci, M. A., Boehnhardt, H., Dotto, E., et al. 2002, A\&A, 392, 335 Barucci, M. A., Belskaya, I. N., Fulchignoni, M., \& Birlan, M. 2005a, AJ, 130, 1291

Barucci, M. A., Cruikshank, D. P., Dotto, E., et al. 2005b, A\&A, 439, L1 Barucci, M. A., Merlin, F., Dotto, E., Doressoundiram, A., \& de Bergh, C. 2006, A\&A, 455, 725

Barucci, M. A., Merlin, F., Guilbert, A., et al. 2008, A\&A, 479, L13 Bonnet, H., Abuter, R., Baker, A., et al. 2004, The Messenger, 117, 17 Brown, M. E., \& Calvin, W. M. 2000, Science, 287, 107

Brown, M. E., Trujillo, C. A., \& Rabinowitz, D. L. 2005, ApJ, 635, L97 Brunetto, R., Barucci, M. A., Dotto, E., \& Strazzulla, G. 2006, ApJ, 644, 646 Buie, M. W., \& Grundy, W. M. 2000, Icarus, 148, 324

Cook, J. C., Desch, S. J., \& Roush, T. L. 2007a, in BAAS, 38, 510

Cook, J. C., Desch, S. J., Roush, T. L., Trujillo, C. A., \& Geballe, T. R. 2007b, ApJ, 663, 1406

Cooper, J. F., Christian, E. R., Richardson, J. D., \& Wang, C. 2003, Earth Moon and Planets, 92, 261

Cruikshank, D. P., Pilcher, C. B., \& Morrison, D. 1976, Science, 194, 835

Cruikshank, D. P., Roush, T. L., Bartholomew, M. J., et al. 1998, Icarus, 135, 389

Dalle Ore, C. M., Barucci, M. A., Emery, J. P., et al. 2009, A\&A, 501, 349 de Bergh, C., Delsanti, A., Tozzi, G. P., et al. 2005, A\&A, 437, 1115 Delsanti, A., Hainaut, O., Jourdeuil, E., et al. 2004, A\&A, 417, 1145 DeMeo, F. E., Fornasier, S., Barucci, M. A., et al. 2009, A\&A, 493, 283 DeMeo, F. E., Barucci, M. A., Alvarez-Candal, A., et al. 2010, in IAU Symp. 263, ed. J. A. Fernández, D. Lazzaro, D. Prialnik, \& R. Schulz, 186 Devillard, N. 1997, The Messenger, 87, 19

Dotto, E., Barucci, M. A., Boehnhardt, H., et al. 2003, Icarus, 162, 408 Dumas, C., Merlin, F., Barucci, M. A., et al. 2007, A\&A, 471, 331 Eisenhauer, F., Abuter, R., Bickert, K., et al. 2003, in SPIE Conf. Ser. 4841, ed. M. Iye, \& A. F. M. Moorwood, 1548

Fornasier, S., Dotto, E., Barucci, M. A., \& Barbieri, C. 2004, A\&A, 422, L43 Fornasier, S., Barucci, M. A., de Bergh, C., et al. 2009, A\&A, 508, 457

Fulchignoni, M., Belskaya, I., Barucci, M. A., de Sanctis, M. C., \& Doressoundiram, A. 2008, in The Solar System Beyond Neptune, ed. M. A. Barucci, H. Boehnhardt, D. P. Cruikshank, \& A. Morbidelli (Tucson, AZ, USA: Univ. of Arizona Press), 181

Gil-Hutton, R., Licandro, J., Pinilla-Alonso, N., \& Brunetto, R. 2009, A\&A, 500, 909

Gladman, B., Marsden, B. G., \& Vanlaerhoven, C. 2008, in The Solar System Beyond Neptune, ed. M. A. Barucci, H. Boehnhardt, D. P. Cruikshank, \& A. Morbidelli (Tucson, AZ, USA: Univ. of Arizona Press), 43

Grundy, W. M., \& Schmitt, B. 1998, J. Geophys. Res., 103, 25809

Guilbert, A., Alvarez-Candal, A., Merlin, F., et al. 2009, Icarus, 201, 272

Hapke, B. 1981, J. Geophys. Res., 86, 3039

Hawarden, T. G., Leggett, S. K., Letawsky, M. B., Ballantyne, D. R., \& Casali, M. M. 2001, MNRAS, 325, 563

Henyey, L. G., \& Greenstein, J. L. 1941, ApJ, 93, 70 
F. E. DeMeo et al.: Spectroscopy of three outer solar system small bodies

Khare, B. N., Sagan, C., Arakawa, E. T., et al. 1984, Icarus, 60, 127

Khare, B. N., Thompson, W. R., Cheng, L., et al. 1993, Icarus, 103, 290

Kouchi, A., \& Kuroda, T. 1990, Nature, 344, 134

Landolt, A. U. 1992, AJ, 104, 340

Lazzarin, M., Barucci, M. A., Boehnhardt, H., et al. 2003, AJ, 125, 1554

Levi, A., \& Podolak, M. 2009, Icarus, 202, 681

Licandro, J., Grundy, W. M., Pinilla-Alonso, N., \& Leisy, P. 2006a, A\&A, 458, L5

Licandro, J., Pinilla-Alonso, N., Pedani, M., et al. 2006b, A\&A, 445, L35

Mastrapa, R. M. E., \& Brown, R. H. 2006, Icarus, 183, 207

Merlin, F., Alvarez-Candal, A., Delsanti, A., et al. 2009, AJ, 137, 315

Moorwood, A., Cuby, J., Biereichel, P., et al. 1998, The Messenger, 94, 7

Owen, T. C., Roush, T. L., Cruikshank, D. P., et al. 1993, Science, 261, 745

Parker, E. N. 1963, Interplanetary Dynamical Processes (New York: Interscience Publishers)

Perna, D., Barucci, M. A., Fornasier, S., et al. 2010, A\&A, 510, A53

Persson, S. E., Murphy, D. C., Krzeminski, W., Roth, M., \& Rieke, M. J. 1998, AJ, 116, 2475

Quirico, E., \& Schmitt, B. 1997, Icarus, 127, 354
Schaller, E. L., \& Brown, M. E. 2007a, ApJ, 670, L49

Schaller, E. L., \& Brown, M. E. 2007b, ApJ, 659, L61

Schmitt, B., Quirico, E., Trotta, F., \& Grundy, W. M. 1998, in Solar System Ices, ed. B. Schmitt, C. de Bergh, \& M. Festou (Kluwer Academic Publishers), 227, 199

Stansberry, J., Grundy, W., Brown, M., et al. 2008, in The Solar System Beyond Neptune, ed. M. A. Barucci, H. Boehnhardt, D. P. Cruikshank, \& A. Morbidelli (Tucson, AZ, USA: Univ. of Arizona Press), 161

Strazzulla, G., \& Palumbo, M. E. 1998, Planetary and Space Science, 46, 1339 Strazzulla, G., \& Palumbo, M. E. 2001, Adv. Space Res., 27, 237

Strazzulla, G., Leto, G., Gomis, O., \& Satorre, M. A. 2003, Icarus, 164, 163

Tegler, S. C., Grundy, W. M., Vilas, F., et al. 2008, Icarus, 195, 844

Trujillo, C. A., Brown, M. E., Rabinowitz, D. L., \& Geballe, T. R. 2005, ApJ, 627,1057

Verbiscer, A., \& Helfenstein, P. 1998, in Solar System Ices, ed. B. Schmitt, C. de Bergh, \& M. Festou (Kluwer Academic Publishers), 227, 157

Zheng, W., Jewitt, D., \& Kaiser, R. I. 2008, arXiv e-prints

Zubko, V. G., Mennella, V., Colangeli, L., \& Bussoletti, E. 1996, MNRAS, 282, 1321 